九州大学学術情報リポジトリ

Kyushu University Institutional Repository

\title{
On the Saturation of the Threshold-type Response in Enzymatic Reaction System
}

Okuyama, Kaoru

Laboratory of Sericultural Chemistry, Faculty of Agriculture, Kyushu University

Okamoto, Masahiro

Laboratory of Sericultural Chemistry, Faculty of Agriculture, Kyushu University

Hayashi, Katsuya

Laboratory of Sericultural Chemistry, Faculty of Agriculture, Kyushu University

https://doi.org/10.5109/23487

出版情報: 九州大学大学院農学研究院紀要. 21 (2/3)，pp.67-77，1977-03. Kyushu University バージョン：

権利関係 : 


\title{
On the Saturation of the Threshold-type Response in Enzymatic Reaction System
}

\author{
Kaoru O kuyama, Masahiro $\mathbf{O}$ kamoto and $\mathrm{K}$ atsuya $\mathrm{H}$ ayashi \\ Laboratory of Sericultural Chemistry, Faculty of Agriculture, \\ Kyushu University 46-02, Fukuoka 812
}

(Received September 29, 1976)

\begin{abstract}
The occurrence of the saturation in a threshold-type response was studied by means of digital simulation using model systems for enzymatic reactions.

The threshold-response did not attain to a saturation level when it was assumed that only the concentration of a product influences the rates of formation and consumption of the substrate. However, the threshold-values and the slopes of the response curves were significantly influenced by the concentration of the product. Saturated thresholdresponses were observed with a model system including a mechanism that the rate of consumption of the substrate was controlled by the concentration of its precursor.
\end{abstract}

\section{INTRODUCTION}

The threshold-type responses in a living organism have attracted attention of many researchers. Ličko (1972) studied an enzymatic model system by means of a steady-state approximation and obtained a threshold-type response which exhibited either an unsaturated or a saturated profile according to selection of rate constants.

In the previous report (Okuyama et al., 1976), a threshold-type response was obtained directly by computation of the rate equations from Ličko's model without any approximation. It was found in the preceding studies that an arbitrary threshold-curve can be connected to a standard curve with the Michaelis constant $(\mathrm{K}$,$) , the threshold-value and the input concentration, and$ that the model system still exhibited the threshold-type response when an oscillating input was given to the system. However, all the computed response did not reach saturation which is thought to be a characteristic of threshold phenomena.

The present study was aimed at establishing a model which is able to produce a saturated threshold-response. In practice, the above mentioned model system was revised stepwise and digital simulations were made at each step to build the desired model system.

\section{COMPUTATION}

Numerical calculations were made about the model system shown in Fig. I-b. The differences of the present system from the previously reported model (Fig. l-a) lie both in an additional subsystem which is connected with a product 


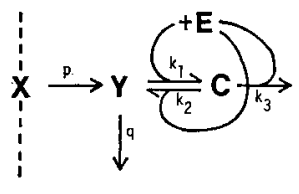

(a)

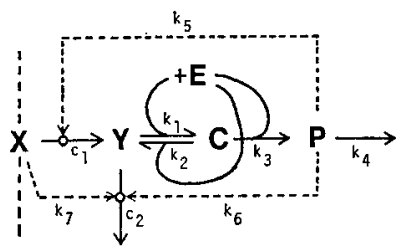

(b)

Fig. 1. Model systems used in the previous study (a) and used in the present study (b).

$\mathrm{P}$, and in the replacement of the previously used rate constants, $p$ and $q$, by new variables, $c_{1}$ and $c_{2}$, respectively.

The rate equations for the system in Fig. l-b may be written as follows:

$$
\begin{aligned}
& \frac{d Y(t)}{d t}=c_{1} X_{0}-k_{1} E(t) Y(t)+k_{2} C(t)-c_{2} Y(t) \\
& \frac{d C(t)}{d t}=k_{1} E(t) Y(t)-k_{2} C(t)-k_{3} C(t) \\
& \frac{d E(t)}{d t}=-k_{1} E(t) Y(t)+k_{2} C(t)+k_{3} C(t) \\
& \frac{d P(t)}{d t}=k_{3} C(t)-k_{4} P(t)
\end{aligned}
$$

Where $X(t)$ is assumed to have the time-invariant-value $X_{0}$ as was in the previous report. The initial concentrations were assumed to be $\mathrm{Y}(0)=\mathrm{C}(0)=$ $P(0)=0$ and $E(0)=c \times 0.2$ (c=l or 2 ).

In order to find a system and sets of parameters which produce a saturated threshold-response on $Y_{\infty}$ value (Y value in steady-state) with input $X_{0}$, simulations were made stepwise in accordance with the revision of the model system as described below.

\section{Step I}

At first it was assumed that the rate coefficients $c_{1}$ and $c_{2}$ were given by the following equation :

$$
\left.\begin{array}{l}
c_{1}=p-k_{5} P(t) \\
c_{2}=q
\end{array}\right\}
$$

Equation (2) means that the $P(t)$ controls the first step $\mathrm{X} \rightarrow \mathrm{Y}$ in a mode of negative feedback with a proportional constant of $k$. . Thus the rate equations for this system may be given by equations (1) and (2). The values of parameters were chosen as $p=0.1, q=1.0, k_{1}=120.0, k_{2}=7.0, k_{3}=5.0, E(0)=0.2$ in arbitrary units, and $K_{n}=0.1$ (the Michaelis constant), $p / q=O .1, k_{3} E(0) / p=10.0$ were used for computation.

From the results of Ličko (1972) and our previous report (Okuyama et al., 1976), the following relationships have been established with respect to the threshold-response for the model system shown in Fig. l-a: 
$\left\{\begin{array}{c}p / q: \text { slope of the idealized }\left(K_{m}=0\right) \text { threshold-curve } \\ k_{3} E(0) / p: \text { threshold-value }\end{array}\right.$

Numerical calculations were performed with changing both $k_{4}$ and $k_{5}$ values. Parameter-sets which had been previously found to give a steady-state were adopted and rate constants $k_{6}$ and $k_{7}$ (shown in Fig. l-b) were not considered yet.

\section{Step II}

From the results of Step I, the variables $c_{1}$ and $c_{2}$ were assumed as follows:

$$
\begin{aligned}
& c_{1}=p-k_{5} P(t) \\
& c_{2}=q+k_{6} P(t)
\end{aligned}
$$

This equation means that the $\mathrm{P}(\mathrm{t})$ controls the formation and consumption of $Y(t)$ by negative and positive feedbacks, respectively. The differential equations for this system may be given by equations (1) and (3). The following values of the parameters were used : p=l. $0, q=0.1, k_{1}=400.0, k_{2}=35.0, k_{3}=5.0, E(0)=O .2$ in arbitrary units, and $K_{m}=0.1, p / q=10.0, k_{3} E(0) / p=1.0$ were used for computation. Simulations were performed with changing the $k_{5}$ and $k_{6}$ values under a fixed value of $k_{4}$.

\section{Step III}

The rate coefficients $c_{1}$ and $c_{2}$ were modified:

$$
\begin{aligned}
& c_{1}=p-k_{5} P(t) \\
& c_{2}=k_{6} q P(t)
\end{aligned}
$$

The second term indicates that the step of $\mathrm{Y}(\mathrm{r})$ consumption is activated linearly by $\mathrm{P}(\mathrm{t})$. The values of the parameters used were the same as those in Step II. Simulations were done stepwise in order to study qualitative relations between one of the additional parameters $\left(k_{4}, k_{5}\right.$ and $k$, ) and the thresholdresponse.

\section{Step IV}

From the results obtained in Step III, the input $X_{0}$ was further assumed to affect the step of $Y(t)$ consumption; thus, $c_{2}$ was defined as follows:

$$
c_{2}=\left(k_{7} X_{0}\right) k_{6} q P(t)
$$

Numerical calculations were performed by changing the values of the parameters included in equation (5).

The values for $k_{1}, k_{2}, k_{3}$ and $E(0)$ used in the present study were chosen from the previously reported parameter-sets, while the values for $p$ and $q$ were changed from the previously reported values.

All the numerical solutions of the differential equations were obtained by means of the MRKGM subprogram (Okamoto et al., 1975) by a FACOM 230-75 digital computer in the Computer Center of Kyushu University.

\section{RESULTS}

\section{Relation between $\boldsymbol{k}_{5}$ and threshold-curve (Step I)}

The relation among $k_{5}, Y_{\infty}, P_{\infty}$, and the input $X_{0}$ is shown in Fig. 2, where 


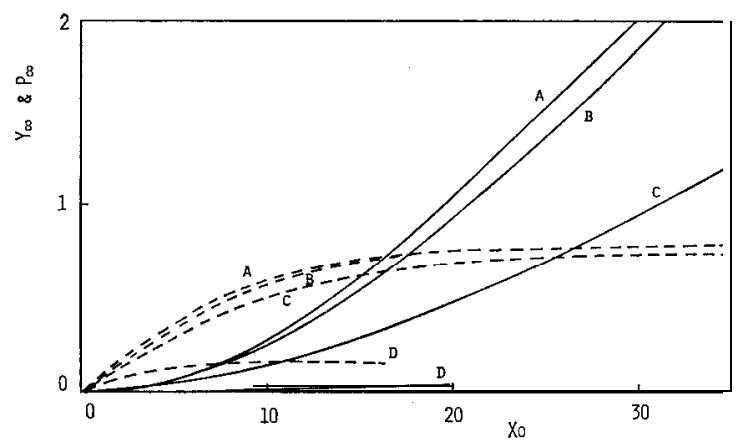

Fig. 2. Computed results in Step I. Solid line is the $Y_{\infty}$ value and broken line is the $\boldsymbol{P}_{\infty}$ value with the changing $k_{5}$ value. Curve $\mathrm{A}, k_{5}=$ 0.001 , curve $\mathrm{B}, k_{5}=0.01$; curve $\mathrm{C}, k_{5}=0.05$; curve $\mathrm{D}, k_{5}=0$. 5. The other parameters were: $p=0.1, q=1.0, k_{1}=120.0, k_{2}=7.0, k_{3}=5.0, k_{4}=1.2$ and $E(0)=0.2$.

$Y_{\infty}$ and $P_{\infty}$ represent the steady-state values of $Y(t)$ and $P(t)$, respectively. Here $k_{4}=1.2$ and the other parameters were set as mentioned above. When $k_{5}=0.001$ was used, the obtained curves are in good agreement with the curve calculated for model l-a because the relations of $k_{5} P(t) \leqslant p$ and $c_{1} \because p$ are realized. When $k_{5}=0.5$ was used, the formation of the substrate $(Y)$ was nearly prevented due to the relationship of $k_{5} P(t) \fallingdotseq p$. As for the curves shown in Fig. $2, P_{\infty}$ increases slightly and takes a steady-state when input $X_{0}$ exceeds a certain limit of its concentration. Consequently, a rate coefficient $c_{1 \infty}=p-k_{5} P_{\infty}$ in the steady-state seems to be held in a constant value, regardless of the amount of input in a region where $P_{\infty}$ appears to be saturated. Adopting an assumption that the tip of each threshold-curve was linear in the region where $P_{\infty}$ shows a steady-value, the slopes of the curves were calculated from Fig. 2. Moreover, assuming that the obtained slopes represented that of idealized threshold-curves, possible threshold-values could be estimated from the figure. Results are shown in Table 1. Because the $K_{m}$ value in this case is equal to 0.1 , practical thresholdvalues must be somewhat larger than estimated threshold-values listed in Table 1. The calculated values for $c_{1} / c_{2}$ and $k_{3} E(0) / c_{1}$ in the steady-state $\left(c_{1 \infty} / c_{2 \infty}\right.$ and $\left.k_{3} E(0) / c_{100}\right)$ are also tabulated.

Table 1. Computed threshold-curves and calculated values at Step I.

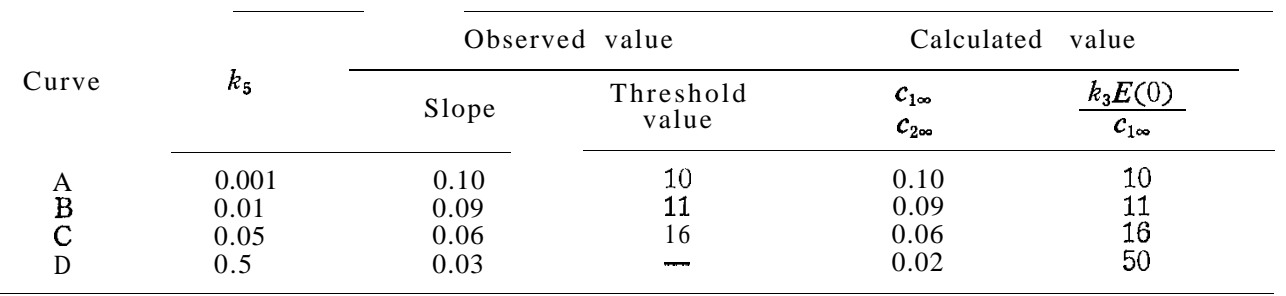




\section{Relation between $\boldsymbol{k}_{4}$ and threshold-curve (Step II)}

In order to obtain curves with steeper slopes than those shown in Fig. 2, p/q ratio (the slope of threshold-curve for the model system shown in Fig. l-a) was enlarged. The values $k_{5}=0.1$ and $k_{6}=0.001$ were fixed and changes of the threshold-curves with parameter $k_{4}$ were studied. Computations were performed only to study whether the curves were saturated or not. As seen in Fig. 3, $P_{\infty}$ values were again saturated and, on the contrary, the threshold-curves for $Y_{\infty}$ were unsaturated. Changes in the threshold-values were not clear from Fig. 3 , though they seem to differ considerably from that of the curve (bold line) for model l-a computed with the same parameters as described above.

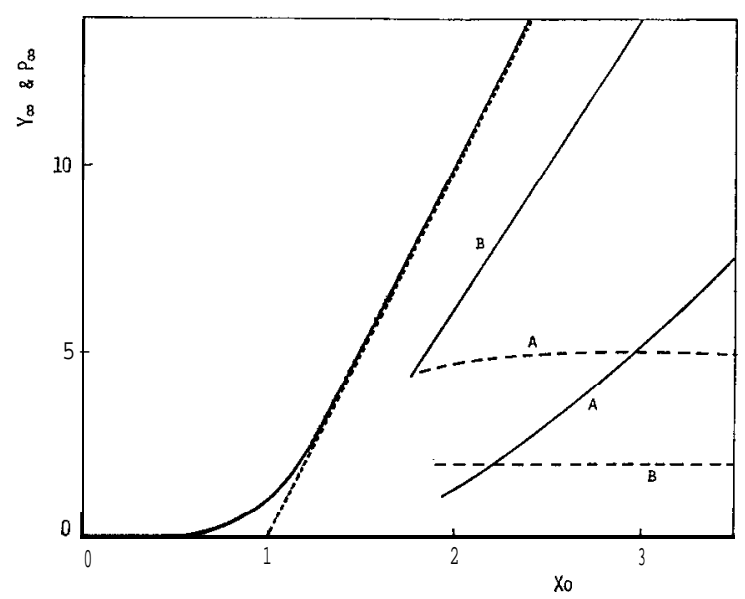

Fig. 3. Computed results in Step II. Solid line is the $Y_{\infty}$ value and broken line is the $P_{\infty}$ value with the changing $k_{4}$ value. Curve A, $k_{4}=0.2$; curve $\mathrm{B}, k_{4}=0.5$. The other parameters were : $\mathbf{p}=\mathbf{l}, \mathbf{0}, q=0 . \mathbf{1}, k_{1}=400 . \mathbf{0}, k_{2}=$ $35.0, k_{3}=5.0, k_{5}=0.1, k_{6}=0.001$ and $E(0)=0.2$. Bold line shows the $Y_{\infty}$ value for model $1-$ a with the same parameter values and dotted line shows idealized threshold-curve.

\section{Relation between $\boldsymbol{k}_{\mathbf{4}}$ and threshold-curve (Step III)}

The values for $p, q, k_{1}, k_{2}$ and $k_{3}$ were the same as in Step II, and $k_{4}=2.0$, $k_{5}=0.1$ and $k_{6}=1.0$ were adopted. In the following studies, these values were defined as a standard set of the parameters (the curve derived from the standard parameter will be called curve S). In relation to the $k_{4}$ value with the standard parameter-set, the behavior of the system was estimated as shown in Fig. 4. The slopes and the threshold-values were estimated in the same manner as described in Step I. As is evident from Table 2, the $k_{4}$ value has a considerable effect on the slopes of the curves but a slight effect on the threshold-values.

\section{Relation between $\boldsymbol{k}_{5}$ and threshold-curve (Step III)}

The dependence of the curve-shape on the change in the $k_{5}$ value in the standard parameter-set is shown in Fig. 5. $\quad P_{\infty}$ took nearly equal values regardless of the $k_{5}$ value and was also saturated. The behavior of the curves except for curve A (this curve cannot be analyzed because its slope is too steep) is 


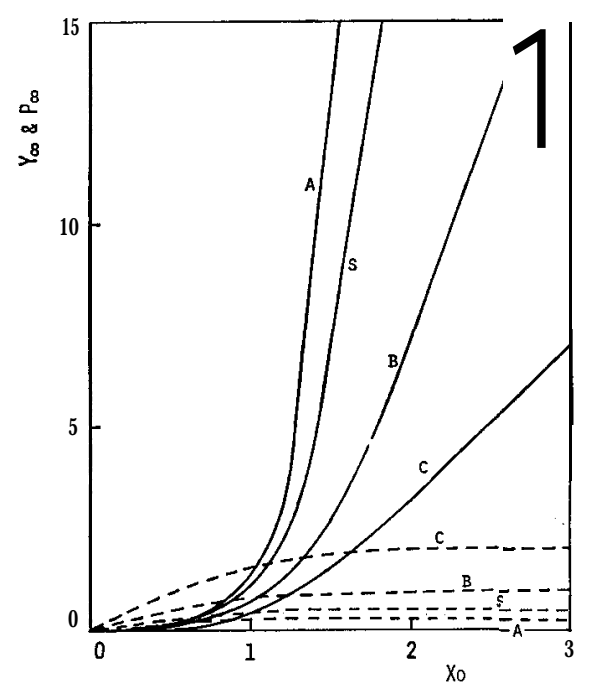

Fig. 4. Computed results in Step III. Solid line is the $Y_{\infty}$ value and broken line is the $P_{\infty}$ value with the changing $k_{4}$ value. Curve $\mathrm{S}, k_{4}=2.0$; curve $\mathrm{A}, k_{4}=3.0$; curve $\mathrm{B}, k_{4}=1.0$; curve C, $k_{4}=0.5$. The other parameters were: $p=1.0, q=0.1, k_{1}=400.0, k_{2}=35.0, k_{3}=5.0, k_{5}=0.1, k_{6}=1.0$ and $E(0)=$ 0.2 .

Table 2. Properties of the computed threshold-curves and calculated values in Fig. 4.

\begin{tabular}{|c|c|c|c|c|c|}
\hline \multirow{2}{*}{ Curve } & \multirow[b]{2}{*}{$k_{4}$} & \multicolumn{2}{|c|}{ Observed value } & \multicolumn{2}{|c|}{ Calculated value } \\
\hline & & Slope & $\begin{array}{c}\text { Threshold } \\
\text { value }\end{array}$ & $\frac{c_{1 \infty}}{c_{2 \infty}}$ & $\frac{k_{3} E(0)}{c_{1 \infty}}$ \\
\hline $\begin{array}{l}\mathbf{S} \\
\mathbf{A} \\
\mathbf{B} \\
\mathbf{C}\end{array}$ & $\begin{array}{l}2.0 \\
3.0 \\
1.0 \\
0.5\end{array}$ & $\begin{array}{r}24 \\
37 \\
11 \\
4\end{array}$ & $\begin{array}{l}1.3 \\
1.4 \\
1.4 \\
1.3\end{array}$ & $\begin{array}{r}19 \\
32 \\
9 \\
4\end{array}$ & $\begin{array}{l}1.1 \\
1.0 \\
1.1 \\
1.3\end{array}$ \\
\hline
\end{tabular}

Table 3. Properties of the computed threshold-curves and calculated values in Fig. 5.

\begin{tabular}{|c|c|c|c|c|c|}
\hline \multirow[b]{2}{*}{ Curve } & \multirow[b]{2}{*}{$k_{5}$} & \multicolumn{2}{|c|}{ Observed value } & \multicolumn{2}{|c|}{ Calculated value } \\
\hline & & Slope & $\begin{array}{l}\text { Threshold } \\
\text { value }\end{array}$ & $\frac{c_{1 \infty}}{c_{2 \infty}}$ & $\frac{k_{3} E(0)}{c_{1 \infty}}$ \\
\hline$\underset{\mathbf{B}}{\mathbf{S}}$ & $\begin{array}{l}0.1 \\
0.2 \\
1: 0\end{array}$ & $\begin{array}{r}24 \\
19 \\
7\end{array}$ & $\begin{array}{l}1.3 \\
1.2 \\
2.1\end{array}$ & $\begin{array}{r}19 \\
18 \\
9\end{array}$ & $\begin{array}{l}1.1 \\
1.1 \\
2.1\end{array}$ \\
\hline
\end{tabular}

shown in Table 3 . Here the threshold-values were influenced considerably by the value of $\boldsymbol{k}_{5}$.

\section{Relation between $\boldsymbol{k}_{6}$ and threshold-curve (Step III)}

The threshold-behavior is shown in Fig. 6 with the changing parameter $k_{6}$ in the standard parameter-set. The values on threshold calculated from the curves are listed in Table 4 . In this case, $\boldsymbol{P}_{\infty}$ was also scarcely influenced by 


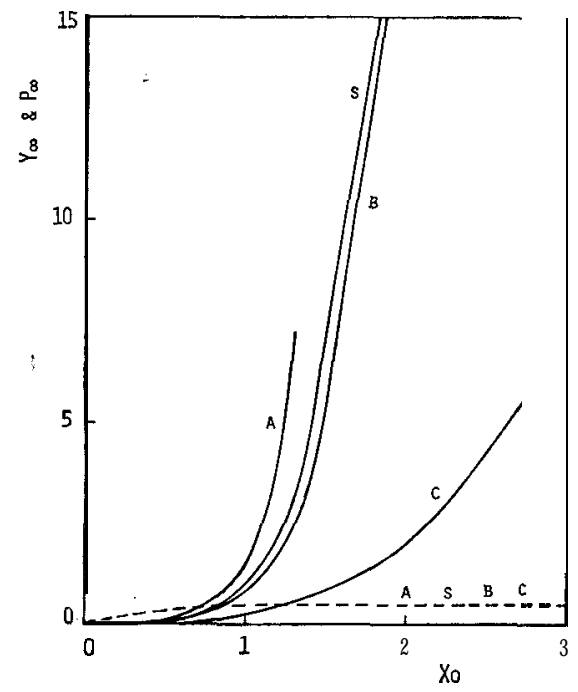

Fig. 5. Computed results in Step III. Solid line is the $Y_{\infty}$ value and broken line is the $P_{\infty}$ value with the changing $k_{5}$ value. Curve $\mathrm{S}, k_{5}=0.1$; curve A, $k_{5}=0.0$; curve $\mathrm{B}, k_{5}=0.2$; curve $\mathrm{C}, k_{5}=1$. 0 . The other parameters w ere: $p=1$. $0, q=0.1, k_{1}=400.0, k_{2}=35.0, k_{3}=5.0, k_{4}=2.0, k_{6}=1.0$ and $\mathbf{E}(0)=$ 0.2 .

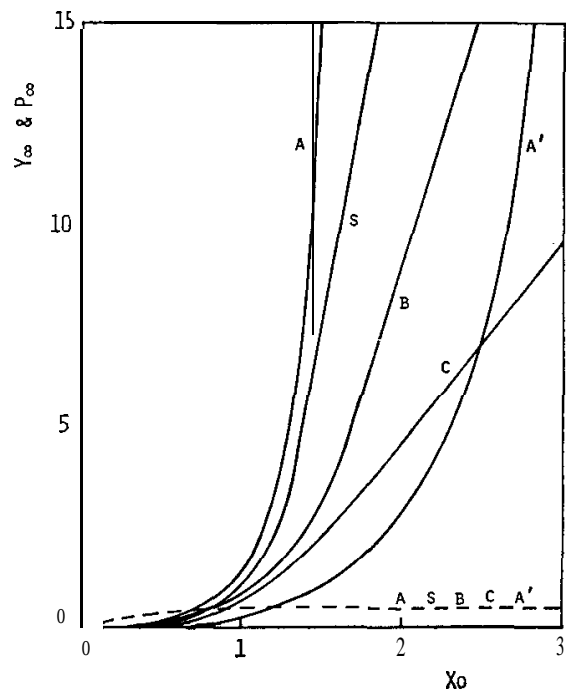

Fig. 6. Computed results in Step III. Solid line is the $Y_{\infty}$ value and broken line is the $P_{\infty}$ value with the changing $k_{6}$ value. Curve $\mathrm{S}, k_{6}=1.0$; curve A, $k_{6}=0.5$; curve $\mathrm{B}, k_{6}=2.0$; curve $\mathrm{C}, k_{6}=4.0$. The other parameters except for curve A' were: $p=1$. $0, q=0.1, k_{1}=400.0, k_{2}=35.0, k_{3}=5.0, k_{4}=$ 2.0, $k_{5}=0.1$ and $E(0)=0.2$. Curve $\mathrm{A}^{\prime}, k_{5}=1.0$ and the other parameters were the same as those of curve A. 
Table 4. Properties of the computed threshold curves and calculated values in Fig. 6.

\begin{tabular}{cccccc}
\hline & & \multicolumn{2}{c}{ Observed value } & \multicolumn{2}{c}{ Calculated value } \\
\cline { 3 - 6 } Curve & $k_{6}$ & Slope & $\begin{array}{c}\text { Threshold } \\
\text { value }\end{array}$ & $\begin{array}{c}\boldsymbol{c}_{1 \infty} \\
c_{2 \infty}\end{array}$ & $\frac{k_{3} E(0)}{c_{1 \infty}}$ \\
\hline $\mathrm{S}$ & 1.0 & 24 & 1.3 & 19 & 1.1 \\
$\mathrm{~A}$ & 0.5 & 36 & 1.3 & 38 & 1.1 \\
$\mathrm{~B}$ & 2.0 & 14 & 1.3 & 10 & 1.1 \\
$\mathrm{C}$ & 1.0 & 4 & 1.0 & 5 & 1.1 \\
$\mathrm{~A}^{\prime}$ & 0.5 & 30 & 2.5 & 10 & $2: 0$ \\
\hline
\end{tabular}

the value of $k_{6}$. The change in the $k_{5}$ value from 0.1 to 1.0 moves curve $\mathrm{A}$ to A', as shown in Fig. 6. As is also evident from curve $\mathrm{C}$ in Fig. 5, the thresholdvalues depend remarkably upon the variation in the $k_{5}$ value.

On the model systems analyzed in Steps I-III, the rate coefficients $c_{1}$ and $c_{2}$ become constant due to the saturation of product amount at steady-state and consequently all the curves have similar shapes to those on model l-a. This is because only the effects of the product concentration have been considered as feedback inputs in Step I-III.

\section{$p / q$ Ratio and threshold-curve (Step IV)}

The threshold-responses calculated with the standard parameter-set and with $k_{7}=1.0$ are shown in Fig. 7. When $p / q$ ratio turned to 20.0 with each of $p$ and $q$ changed, the profile of response changed profoundly as shown in Fig. 8. Curves A and B showed a clear threshold-nature. The threshold-value in model l-a with $p=2.0$ turned to 0.5 and this relation was also held in curve A in Fig. 8. The curves obtained here may be considered to be practically saturated.

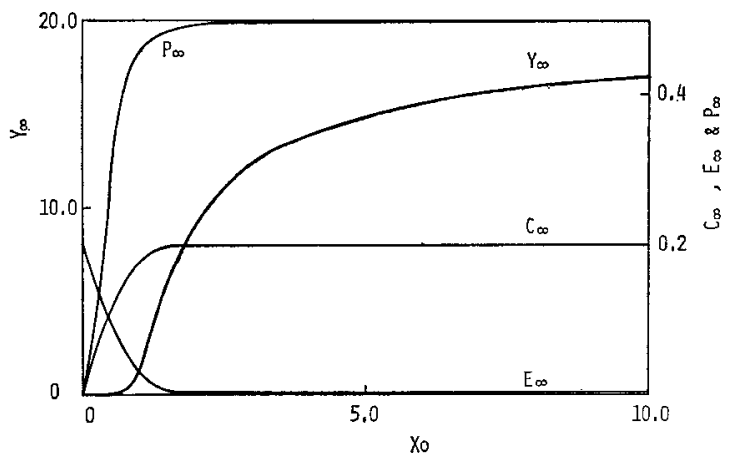

Fig. 7. Computed results in Step IV for the standard parameter values: $p=1.0, q=0.1, k_{1}=400.0, k_{2}=35.0, k_{3}=5.0, k_{4}=2.0, k_{5}=0.1, k_{6}=1.0, k_{7}=1.0$ and $E(0)=0.2$.

\section{$\boldsymbol{E}(\mathbf{0})$ and threshold-curve (Step IV)}

Curve C (in Fig. 9) was obtained when only the initial concentration $E(0)$ of curve $\mathrm{A}$ in Fig. 8 was changed from 0.2 to 0.4 . Figure 9 also depicts the relation among curves $\mathrm{C}, \mathrm{A}$ and the standard curve $\mathrm{S}$. The curves $\mathrm{A}$ and $\mathrm{S}$ are transcribed from Fig. 8. As a result of the change in the $E(0)$ value, $k_{3} E(0) / p$ 


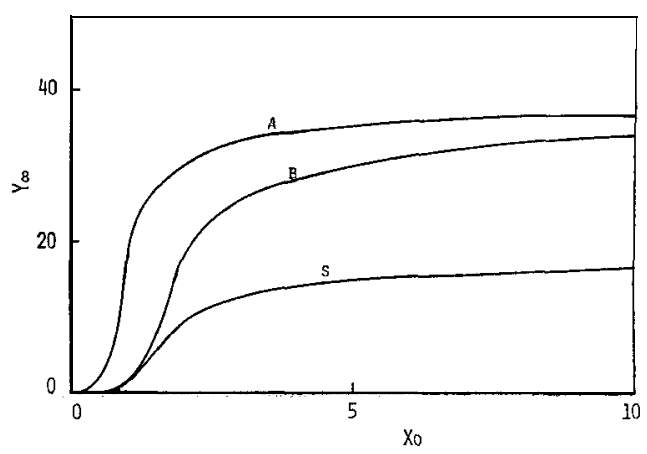

Fig. 8. Computed results in Step IV with the changing $\mathbf{p} / \mathbf{q}$ ratio. Curve S, $p=1.0$ and $q=0.1$; curve A, $p=2.0$ and $q=0.1$; curve B, $p=1.0$ and $q=$ 0.05 .

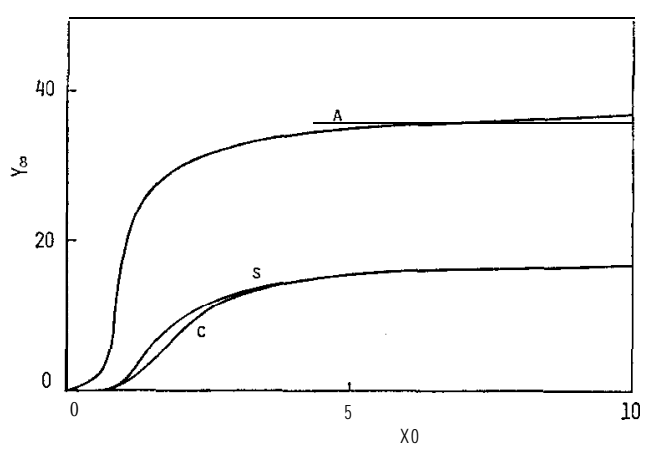

Fig. 9. Computed results in Step IV with the changing $\mathbf{E}(\mathbf{0})$ value. Curves $\mathrm{S}$ and $\mathrm{A}$ are the same as in Fig. 8. Curve $\mathrm{C}, p=2.0, q=0.1, E(0)=0.4$ and the other parameters were the same as those of curve $S$.

returns to its original value of 0.1 . In relation to this, curve $\mathrm{C}$ exhibits a larger threshold-value than curve A. Furthermore, curve C seems to have the same saturated concentration as the standard curve $S$ has, although their shapes are slightly different from each other. Moreover, the ratios of $P_{\infty}$ to $p$ in curves $\mathrm{A}$ and $\mathrm{S}$ are evenly $1 / 2$, and the value of $k_{3} E(0) / c_{1 \infty}$ is about 1.0 for each curve; this value coincides with the threshold-value estimated from the figure.

\section{Relation between $\boldsymbol{k}_{7}$ and threshold-curve (Step IV)}

The changes of curve B (Fig. 8) in relation to the changes of the $k_{7}$ value are shown in Fig. 10. The threshold-values for the four curves are exactly the same and $P_{\infty}$ also took an equal value for the four curves. The value of $k_{3} E(0) / c_{1 \infty}$ is about 1.1 for the four curves and is in agreement with the threshold-value estimated from the figure. It is clear from the assumption on $c_{1}$ and $c_{2}$ that curve $\mathrm{F}$ coincides with curve $\mathrm{S}$ in Fig. 9 .

In Step IV, the effect of an additional feed-forward subsystem, which consumes the substrate, on the threshold-response was evidenced clearly. Further- 


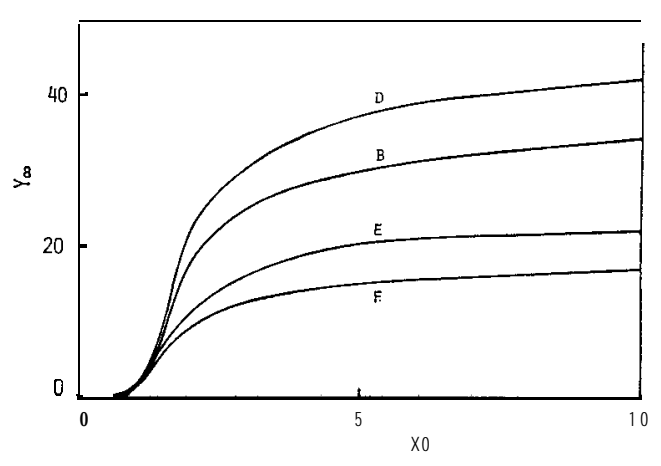

Fig. 10. Computed results in Step IV with the changing $k_{7}$ value. Curve $\mathrm{B}, k_{7}=0.8$; curve $\mathrm{D}, k_{7}=1$. 0 ; curve $\mathrm{E}, k_{7}=1.5$; curve $\mathrm{F}, k_{7}=2.0$. The other parameters were the same as curve $B$ in Fig. 8 .

more, it was found that the threshold-curves obtained were all saturated as well as the values of $P_{\infty}$ and $C_{\infty}$.

\section{DISCUSSION}

As for Steps I-III, and in a region where the coefficients $c_{1}$ and $c_{2}$ can be assumed to be constant, the slope of the response curve plotted in the figures was nearly the same as the value of $c_{1 \infty} / c_{2 \infty}$ as shown in Tables 1-4. This was easily expected because the $c_{1} / c_{2}$ value corresponds to $p / q$, which is the slope of the idealized threshold-curve based on model l-a. When the $c_{1} / c_{2}$ value is approximated by $c_{1 \infty} / c_{2 \infty}$, the practical slope of the curve with $K_{m}=0.1$ may be somewhat more gentle than the $c_{1 \infty} / c_{2 \infty}$ value since the curve with $K_{m}=0.1$ shows an exponential shape around the threshold-value of $X_{0}$.

Since $P_{\infty}$ is related to both $c_{1 \infty}$ and $c_{2 \infty}$, an error on $P_{\infty}$ makes a considerable error on the $c_{1 \infty} / c_{2 \infty}$ value. The slopes obtained from the curves in Figs. 2,4,5 and 6 , therefore, also contain noticeable error. Thus, it can be said that the value $c_{1 \infty} / c_{2 \infty}$ is practically equal to the slope of the idealized threshold-curve for model l-b under the conditions used for the present investigation.

On the other hand, the threshold-value obtained by extrapolation of the response curve and the calculated value of $k_{3} E(0) / c_{10}^{*}$ showed an inverse relationship in their order of magnitude and there seems to be no correlation between both values.

The saturated threshold-curves first appeared in Step IV. Here, $c_{1}$ and $c_{2}$ were influenced by the value of $P$ and $c_{2}$ still depends on the magnitude of input. The relation between the $p / q$ ratio and the initial slope of the response curve, therefore, becomes very complicated. For curves $\mathrm{S}$ and $\mathrm{C}$, the relation was inverted due to the difference in the initial concentration of enzyme. From Figs. 5 and 8, it is obvious that the saturation still appeared under the conditions of $k_{5}=0$, implying that the saturation of response may be reached in the ab-

* This value is corresponding to the threshold-value of model 1-a. 
sence of any feedback subsystem from the product if only suitable values for the other parameters were selected. This fact suggests that the saturated response will be produced by more simple model systems than those subjected to the present analysis.

In the present study, only qualitative relationships between the saturated response and the structure of enzymatic reaction system were investigated. Quantitative relationships may be searched by a more detailed computational study. Nevertheless, it will be valuable that the present study pointed out the possibility that a relatively simple enzyme system will be able to realize a saturated threshold-response.

Other problems yet to be solved in future are to establish model systems that can produce a stepped threshold-curve and to clarify the molecular mechanism by which the activation of the substrate consumption process by the input takes place, as seen in Step IV.

\section{REFERENCES}

Ličko, V. 1972 Some biochemical threshold mechanisms. Bull. Math. Biophysics, 34: 103112

Okamoto, M., Y. Aso, D. Koga and K. Hayashi 1975 Note on steady-state approximation of enzyme kinetics. J.Fac. Agr., Kyushu Univ., 19: 125-138

Okuyama, K., M. Okamoto, Y. Aso and K. Hayashi 1976 Studies on the threshold mechanism of enzymatic reaction. J. Fac. Agr., Kyushu Univ., 20: 87-95 